\title{
Qualitative and quantitative analysis of emergency department cardiac arrest publications
}

\author{
Hsiang-Yun Lo ${ }^{1,2}$, Shang-Kai Hung ${ }^{1}$, Chip-Jin Ng ${ }^{1}$, Shou-Yen Chen ${ }^{1,3, *}$
}

\author{
${ }^{1}$ Department of Emergency Medicine, \\ Chang Gung Memorial Hospital and \\ Chang Gung University, 333 Taoyuan, \\ Taiwan \\ ${ }^{2}$ Institute of health policy and \\ management, National Taiwan \\ University, 106 Taipei, Taiwan \\ ${ }^{3}$ Graduate Institute of Clinical Medical \\ Sciences; Division of Medical Education, \\ College of Medicine, Chang Gung \\ University, 333 Taoyuan, Taiwan

*Correspondence
allendream0621@yahoo.com.tw;
allendream0621@gmail.com;
8902007@cgmh.org.tw
(Shou-Yen Chen)

\begin{abstract}
Cardiac arrest is a medical emergency with a poor prognosis. Patient characteristics and outcomes are associated with location and are traditionally categorized into out-ofhospital cardiac arrest (OHCA) or in-hospital cardiac arrest (IHCA). Increasing evidence has revealed that cardiac arrest occurring in the emergency department is distinct from OHCA or IHCA in other locations in hospitals, but most academic publications combine these populations and apply the knowledge arising from OHCA or IHCA to patients with emergency department cardiac arrest (EDCA). The aim of this study was to identify the research direction of EDCA in the past 20 years and to analyze the characteristics and content of academic publications. We searched the MEDLINE and EMBASE databases for eligible articles until May 30, 2021. Two independent reviewers extracted data by using a customized form to record crucial information, and any conflicts between the two reviewers were resolved through discussion with another independent reviewer. The aggregated data underwent a scoping review and analyzed qualitatively and quantitatively. In total, 52 original articles investigating EDCA were included; only 15 articles simply focused on EDCA, while other articles involved OHCA or IHCA simultaneously. There were 3 articles discussing the relationship of overcrowdedness and EDCA, 12 articles for prediction and risk factors associated with EDCA, 15 articles for epidemiology and prognosis, and 22 articles for specific diagnostic or resuscitation skills with regard to EDCA. Studies focusing on EDCA are increasing but still scarce. Applying the knowledge arising from OHCA or IHCA to EDCA is questionable, and research focused on EDCA is necessary. ED overcrowdedness-associated EDCA and prediction models for EDCA are essential topics that need further investigation.
\end{abstract}

\section{Keywords}

Emergency department cardiac arrest; Resuscitation; In-hospital cardiac arrest; Overcrowdedness

\section{Introduction}

Cardiac arrest is a medical emergency characterized by cessation of cardiac mechanical activity with the absence of signs of circulation [1]. The outcome of cardiac arrest is poor despite considerable efforts on treatment in recent decades. Survival varies according to the location of the cardiac arrest occurred and the associated critical interventions [2]. Cardiac arrest is traditionally categorized into out-of-hospital cardiac arrest (OHCA) and in-hospital cardiac arrest (IHCA), which are considered as two distinct groups with different characteristics and prognoses. IHCA has a higher prevalence of a reversible etiology, higher short-term and long-term outcomes and a better cerebral performance category upon survival [3].

EDCA is traditionally classified as part of IHCA, a United Kingdom national database research in 2014 revealed EDCA patients comprised up to $18.2 \%$ of all IHCA cases [4]. In 2008, Kayser et al. [5] published an article demonstrating that EDCA has unique characteristics and better survival and neurologic outcomes than cardiac arrest events in other hospital locations. Further studies also support the findings and found that a better outcome of EDCA may contribute to the close monitoring of vital signs to detect deterioration early, the earlier delivery of advanced cardiac life support and the resuscitation experience of health providers in the ED [6].

Understanding the academic publication trend of EDCA is crucial for further investigation into this unique population. The aim of this study was to identify the research direction of EDCA in the past 20 years, demonstrate a scoping review and to analyze the characteristics and content of academic publications. Our study may help to identify and map current available evidence on EDCA and facilitate further research.

\section{Materials and methods}




\subsection{Scoping review protocol}

A scoping review was conducted based on a predesigned protocol in accordance with the Preferred Reporting Items for Systematic Reviews and Meta-Analyses (PRISMA) extension for scoping reviews [7].

\subsection{Search strategy}

We searched the MEDLINE and EMBASE databases for eligible articles until May 30, 2021, with the most recent search on June 01, 2021. The search strategy was based on the following algorithm: ("Cardiac arrest" [All Fields] OR "Cardiopulmonary resuscitation" [All Fields]) AND ("Emergency room" [All Fields] OR "Emergency department" [All Fields]). We also supplemented the articles by the "snowball method" and manually browsed the reference lists of all included articles for additional eligible studies.

\subsection{Inclusion and exclusion criteria}

We aimed to include all original articles discussing cardiac arrest in the emergency department. EDCA was defined as cardiac arrest initially occurred in emergency department and need interventions including resuscitation and advanced cardiac life support. Articles focused on OHCA or cardiac arrest occurring in other units in the hospital (ward, ICU, operation room, and postanesthesia care unit) were excluded. We also excluded articles discussing IHCA but did not separate EDCA as a group. We excluded non-original articles, such as reviews, editorials, perspectives, short or special communications, and letters to editors. Furthermore, articles not utilizing the English language were excluded.

\subsection{Study selection and data charting}

Fig. 1 depicts the study selection and review processes. After selecting articles from two databases, we manually removed duplicate articles. Two independent reviewers (HYL and SKH) scanned the titles and abstracts of all articles to determine relevancy in light of the inclusion/exclusion criteria. Articles without abstracts were included for full-text assessment and evaluated at that stage. Two reviewers shared their results after scanning the titles and abstracts of all articles, and only articles that were excluded by both reviewers were eliminated from further full-text assessment. During the fulltext assessment stage, we used a customized Excel sheet to record the essential information of the article. Article type, first author of the article, publication journal, publication date, included group of cardiac arrest, and major objective of the article were extracted. Then, two reviewers evaluated the extracted full-text articles separately according to the criteria. Any conflicts between the two reviewers regarding the extracted articles were resolved through discussion with another reviewer (SYC). Two reviewers also searched additional eligible studies from the reference lists of all included articles by the "snowball method". The included articles were further quantitatively analyzed or described narratively.

\subsection{Statistical analysis}

Descriptive statistics of aggregated data are presented using counts and proportions. We performed analysis for publication time, target research group and the study objective of the article. Statistical analysis was performed using Microsoft Excel software (2016, Microsoft Corporation, Seattle, Washington, USA).

\section{Results}

The search yielded 2653 and 3573 articles from MEDLINE and EMBASE, respectively. After removing duplicates, 2998 articles remained; their titles and abstracts were scanned, and 86 relevant articles were identified. The full texts of these 86 articles were further reviewed, after which 48 articles were included. An additional 4 articles were supplemented by the "snowball method", and a total of 52 articles were included for data extraction. The key information of the 52 articles is summarized in Supplementary Table 1, with the articles presented in alphabetical order by name of first author.

Of the 52 original articles, 25 articles included OHCA and EDCA groups, 15 articles included an EDCA group only, 11 articles included both EDCA and IHCA groups and 1 article included OHCA, EDCA and IHCA groups (Fig. 2). The publications involving cardiac arrest increased with time, especially articles focusing on EDCA (Fig. 3). The topics of the articles are presented in Fig. 4. There were 3 articles discussing the relationship of overcrowdedness and EDCA, 12 articles discussing the prediction and risk factors related to EDCA, 15 articles discussing the epidemiology and prognosis of EDCA and 22 articles discussing the specific diagnostic or resuscitation skills related to EDCA.

We summarized the major characteristics of articles of prediction using methods of machine learning or scoring systems in Table 1. The major aim and the performance of the best predictor in the research were listed. Table 2 shows the articles discussing clinical skills including ultrasound, periintubation procedures, extracorporeal CPR and targeted temperature management in ED, and the major findings are summarized.

\section{Discussion}

Our study is the first to review and analyze published academic articles focusing on cardiac arrest in the ED. Since Kayser published a study in 2008 demonstrating the unique features of EDCA and suggested separating EDCA as an independent group, we found that publications focusing solely on EDCA increased [5]. Although increasing evidence has revealed that different cardiac arrest groups have diverse characteristics and represent different populations, only a small portion of the included studies focused on EDCA specifically. Due to the heterogeneity of the research target group, the results of the articles may obscure the potential findings. Increasing studies focusing on EDCA have been noted in recent years, and further research may obtain more specific findings for patients with EDCA. We adopted scoping review but not systematic review because EDCA is an emerging concept with several knowledge gaps. Rather than answering a particular clinical question, the 


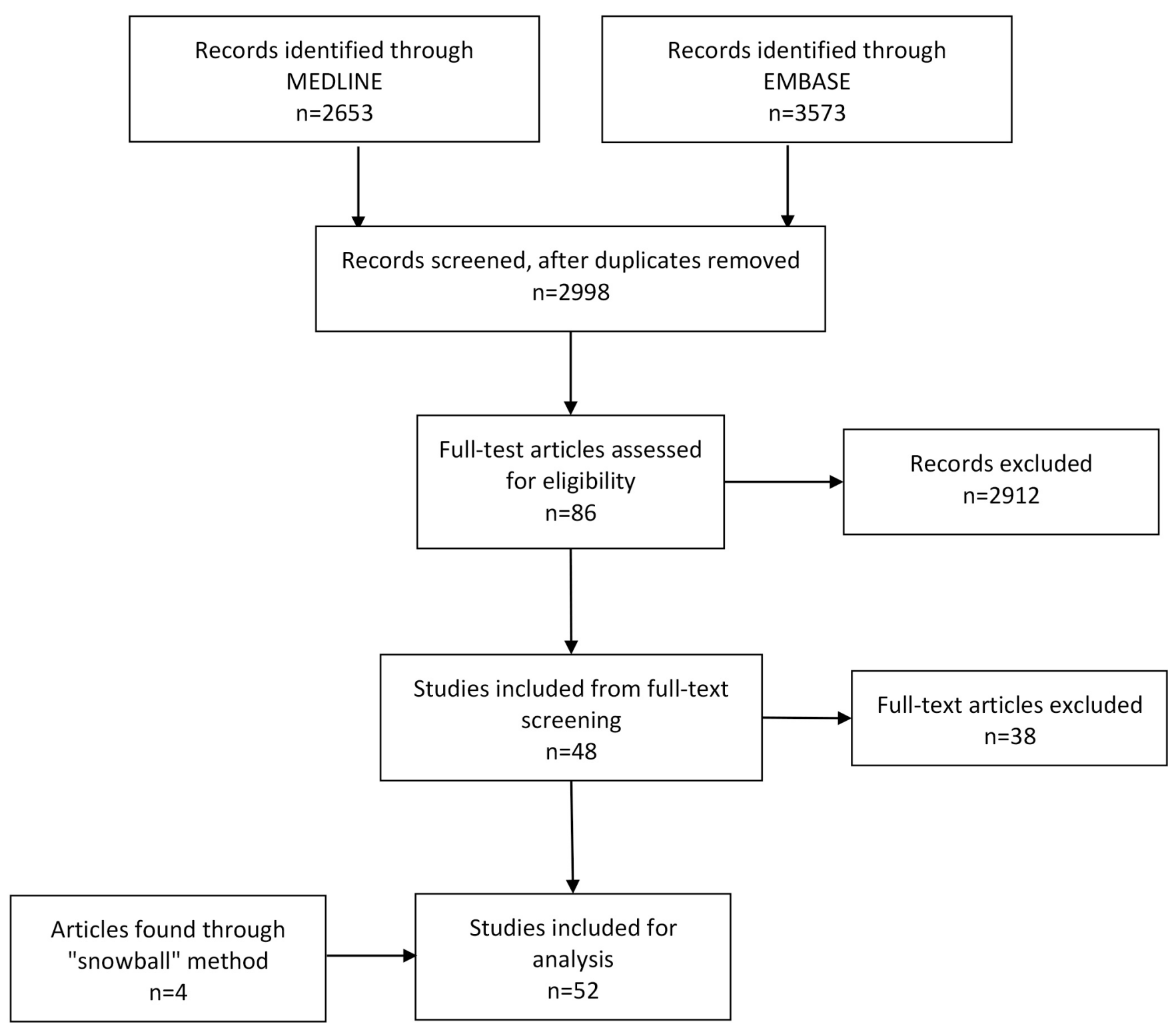

F I G U RE 1. PRISMA flow diagram of the study selection process.

\section{Included CA groups of articles}

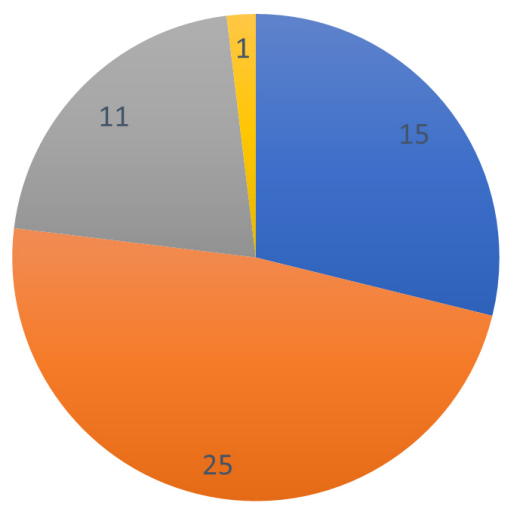

EDCA OHCA+EDCA EDCA+IHCA OHCA+EDCA+IHCA

F I G U RE 2. Included cardiac arrest groups of articles. major aim of this article is to identify and map current available evidence. With this purpose, scoping review will be a better approach [8].

\subsection{Epidemiology and prognosis}

EDCA accounts for 12-20\% of all cardiac arrest events within hospitals and has better survival and neurological outcomes than cardiac arrest occurring in other locations in hospitals. The overall survival to discharge is $22.2-48.1 \%$, and patients in this group have a higher prevalence of cardiac etiology as the precipitating event than other cardiac arrest groups [5, 9-11]. A nationwide registry-based study found that the witnessing status of cardiac arrest is an important factor for prognosis [2]. This may be a possible reason for the better prognosis of patients with EDCA since there was full-time, on-site accessibility of health professionals at the ED. Better experiences of health professionals treating patients with cardiac arrest may also contribute to the better prognosis of EDCA [12]. 
TA B L E 1. Key information of articles focused on prediction.

A rise in peri-arrest MEWS reduced the chance of survival to discharge by 0.77 fold (95\% CI: $0.60-0.97, p=0.028)$

19 Use of the National Early Warning Score for predicting in-hospital Using NEWS to predict cardiac arrest in older adults mortality in older adults admitted to the emergency department

NEWS, AUROC $=0.820$ (95\% CI: 0.806 $0.833)$

41 Emergency Department Triage Early Warning Score (TREWS) predicts To evaluate different scoring systems in predicting in-hospital mortality in the emergency department

cardiac arrest

TREWS, AUROC $=0.906(95 \%$ CI: $0.903-0.908)$

48 Validation of different score systems in predicting cardiac arrest occurrence of ST-elevation myocardial infarction

To evaluate different scoring systems in predicting cardiac arrest in STEMI patients

6-month GRACE score, AUC $=0.727$ (95\% CI: 0.645-0.809)

49 Predictors of in-hospital cardiac arrest within $24 \mathrm{~h}$ after emergency Using NEWS and other factors to predict 24-h department triage: A case-control study in urban Thailand cardiac arrest $=0.91$ (95\% CI: 0.89-0.93)

Machine learning

10 Developing neural network models for early detection of cardiac arrest in emergency department

29 Prediction of cardiac arrest in critically ill patients presenting to the emergency department using a machine learning score incorporating heart rate variability compared with the modified early warning score

32 Prediction of adverse cardiac events in emergency department patients with chest pain using machine learning for variable selection

Using ANN classifier to predict 24-h cardiac arres event

Hybrid ANN classifier, AUROC $=0.936$ (95\% CI: 0.933-0.939)

Using machine learning incorporating with HRV to predict $72-\mathrm{h}$ cardiac arrest event in critically ill patients

Using clinical signs and HRV machine learning score to predict 72-h MACE

45 Prediction of Cardiac Arrest in the Emergency Department Based on Machine Learning and Sequential Characteristics: Model Development and Retrospective Clinical Validation Study

47 Manifold ranking based scoring system with its application to cardiac arrest prediction: A retrospective study in emergency department patients

Using machine learning and sequential characteristics to predict cardiac arrest event

Using clinical signs and HRV manifold rankingbased machine learning to predict $72-\mathrm{h}$ cardiac arrest
Machine learning score, AUROC $=0.781$

Machine learning score with top three variables, AUROC $=0.812(95 \% \mathrm{CI}$ : 0.716-0.908)

RF-based model with a $10 \%$ balancing ratio, $\mathrm{AUROC}=0.97$ (95\% CI: 0.97-0.97)

Proposed scoring system on balanced dataset, AUROC $=0.907$; imbalanced dataset, $\mathrm{AUROC}=0.774$

ANN, artificial neural network; RF, random forest; MEWS, Modified Early Warning Score; CI, confidence interval; GRACE, Global Registry of Acute Coronary Event; NEWS, National Early Warning Score; TREWS, Emergency Department Triage Early Warning Score.

*factors including: higher initial NEWS at triage, lack of improvement in the NEWS, having CAD as a comorbid disease, the use of a vasoactive agent, an initial serum bicarbonate level lower than $23.5 \mathrm{mmoL} / \mathrm{L}$. 


\section{Time distribution of included articles}

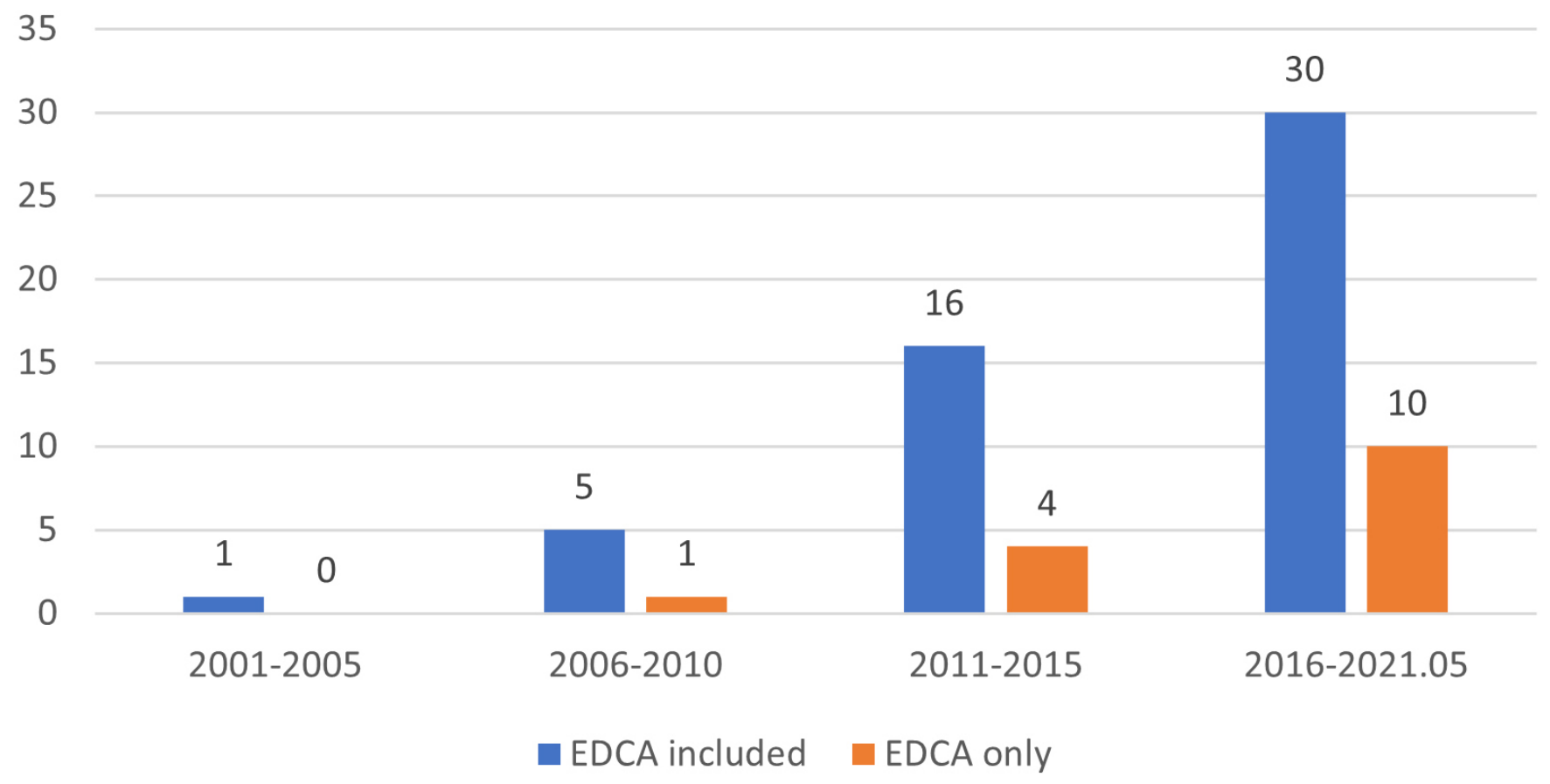

F I G U R E 3. Time distribution of included articles.

\subsection{Overcrowdedness and EDCA}

Chang et al. [13] published an article demonstrating that ED overcrowdedness is associated with an increased incidence of EDCA. The parameter for ED overcrowdedness in this article is defined by the ratio of the number of beds occupied by patients to the total number of licensed ED beds [13]. However, another study using the same parameter revealed a conflicting result [14]. Kim et al. [15] used another parameter and demonstrated that the ratio of the total number of ED patients to the number of beds in the ED was positively correlated with EDCA occurrence. Other than the ED bed number to patient ratio, Tsai et al. [16] adopted the patient-to-ED staff ratio to indicate ED overcrowdedness and proved that a higher patientto-nurse ratio was associated with an increase in the incidence of EDCA. A systematic review study in 2011 identified 71 parameters indicating ED overcrowdedness and the authors found there were no objective criterion standard [17]. Based on the input-throughput-output conceptual model, various causes may affect ED overcrowdedness, but these factors could vary based on different health care systems, community characteristics and hospital determinants [18]. A single, universal index representing ED overcrowdedness may be impossible for the above reasons, so developing a multifactorial, dynamic, and perhaps individualized index would be more feasible [19].

\subsection{Prediction and risk factors}

\subsubsection{Scoring system}

A scoring system has been used to predict EDCA occurrence. Wang et al. [20] demonstrated that the pericardiac arrest modified early warning score is an independent predictor of the chance of survival to discharge in patients with EDCA. Several studies have evaluated the National Early Warning Score (NEWS) to predict cardiac arrest in patients admitted to the ED. Kim et al. [21] demonstrated that the NEWS at ED triage can predict further IHCA occurrence in patients older than 65 years. Srivilaithon et al. [22] combined triage NEWS with four other predictors and had better performance than NEWS alone in predicting EDCA. Lee et al. [23] developed a new scoring system, the Triage in Emergency Department Early Warning Score (TREWS), based on the foundation of the NEWS, which had a better performance than the NEWS, MEWS and Rapid Emergency Medicine Score. These scoring systems used in the ED shared few characteristics. First, the composition of the scoring system is mostly based on simple vital signs and can be easily assessed in ED settings. Second, the formula can be calculated easily and integrated into a modern electronic health record system. Third, the scoring system can be monitored closely and dynamically. However, the real impact of the scoring system on the clinical setting is unknown, and the major concern is the practicality of the scoring system beyond the clinical judgment for physicians. The clinical impact of the scoring system on the current setting should be further evaluated prospectively.

\subsubsection{Machine learning}

Machine learning algorithms are an objective, replicable approach to integrate multiple variables, and they have shown promise to improve diagnostic ability in different conditions [24-27]. The advantage of machine learning is the ability to process complex nonlinear relationships in the data and yield more stable predictions. Along with the prominent develop- 
Category of the included articles

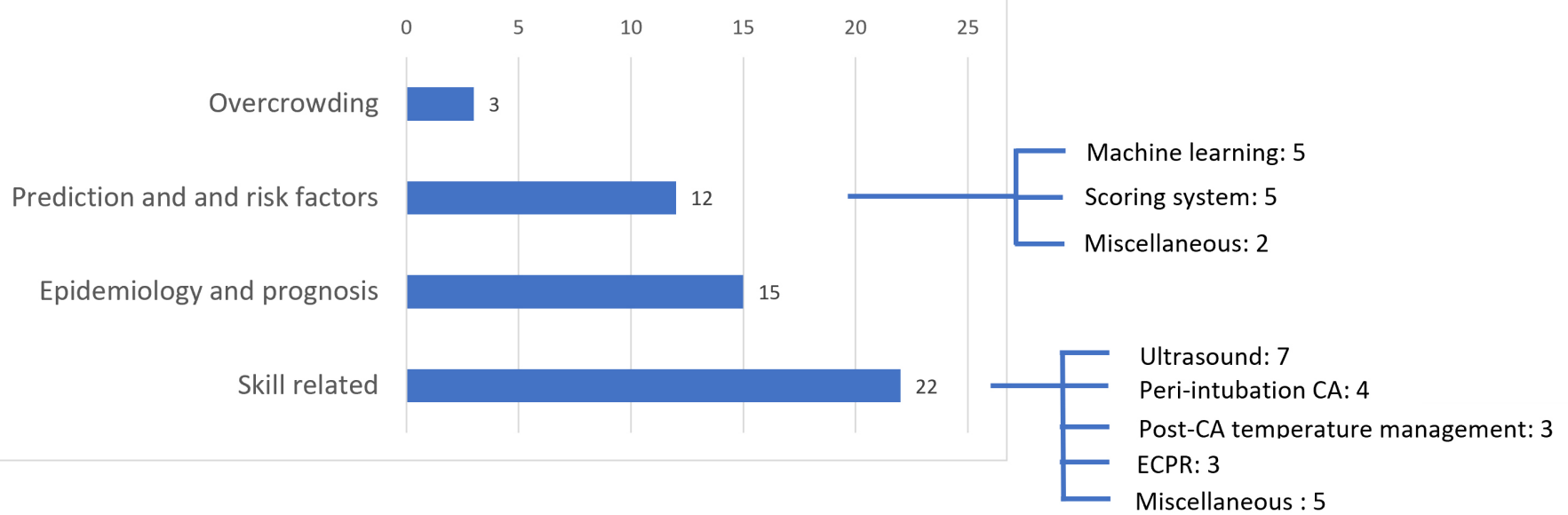

F I G U RE 4. Category of included articles.

ment in health informatics and rapid advances in computer processing techniques, the application of machine learning in emergency medicine has appeared. As a result, associated studies have also bloomed in the past 10 years [28]. Hock Ong et al. [29] validated a machine learning algorithm incorporating heart rate variability (HRV) and proved better performance than the MEWS in predicting EDCA. Liu et al. [30] developed a random forest-based machine learning algorithm and proved good performance when predicting major adverse cardiac events, including cardiac arrest, among patients admitted to the ED with chest pain. They also developed a novel machine learning algorithm based on semi-supervised manifold ranking to predict EDCA [31]. Other studies also showed a unique prediction model for EDCA occurrence [32]. Although there are many advantages to machine learning algorithms, their application has some challenges. First, machines learn from examples rather than being programmed with rules; thus, the performance and ability of machines to learn is driven by the quality of data provided, which is why machine learning systems are so-called data-intensive systems. A central challenge in building a machine learning model is assembling a representative, diverse dataset [33]. Second, the more complex the prediction model is, the harder it is to interpret clinically, and such a "black box" decision-making pattern will present obstacles in clinical utility. Third, the consequences of integrating and applying the results from machine learning systems into real ED workflows are unknown and still need further investigation.

\subsection{Skills applied in EDCA}

\subsubsection{Ultrasound}

The included studies associated with ultrasound evaluated the impact of ultrasound-assisted resuscitation and the barriers to performing ultrasound in patients with cardiac arrest. Specific ultrasound findings in patients with cardiac arrest and their correlation with outcome were also mentioned. However, these articles studied all patients with cardiac arrest without distinguishing subgroups, and the study focusing on ultrasound application in patients with EDCA is surprisingly lacking [34].
Increasing evidence has revealed different patient characteristics, major contributing causes of cardiac arrest and resuscitation response times between OHCA and EDCA, so the application of ultrasound in patients with EDCA may need further study $[35,36]$.

\subsubsection{Peri-intubation cardiac arrest}

Cardiac arrest is a notorious fatal complication of emergent airway management in the ED. The incidence is $0.5-4.2 \%$ and is associated with peri-intubation conditions, procedural processes and pharmacological effects of rapid sequential intubation [37]. The occurrence of peri-intubation cardiac arrest has increased in-hospital mortality, so prevention is important [38]. These studies identified possible risk factors associated with peri-intubation cardiac arrest, including periprocedural hypotension, critical hypoxemia, inadequate time for full preparation, multiple intubation attempts and underlying pulmonary diseases. Identifying the risk factors to prevent cardiac arrest during intubation should be noted. Hoehn et al. [39] advocated creating a rapid reaction team to reduce the risk of peri-intubation cardiac arrest among highrisk patients and successfully developed a novel approach to mitigate the risk for peri-intubation cardiac arrest in pediatric patients. This approach may need to be validated in adult patients for further application.

\section{Limitation}

This study was not without limitations. First, some studies discussing IHCA did not specifically define the location of events, and these articles were not included in this article, which may contribute to potential selection bias. Second, we only included articles published in English, and some important information published in different languages may have been overlooked. Third, we only searched two datasets. Although we adopted the "snowball" method for a more comprehensive search, we believe that some publications have not been included. 
TA B L E 2. Key information of articles focused on ultrasound and peri-intubation cardiac arrest.

15 Rhythms and prognosis of patients with cardiac arrest, emphasis on pseudo-pulseless electrical activity: another reason to use ultrasound in emergency rooms in Colombia

17 A description of echocardiography in life support use during cardiac arrest in an Emergency Department before and after a training program

27 Barriers to point-of-care ultrasound utilization during cardiac arrest in the emergency department: a regional survey of emergency physicians

30 Outcome in cardiac arrest patients found to have cardiac standstill on the bedside emergency department echocardiogram

37 Do Electrocardiogram Rhythm Findings Predict Cardiac Activity During a Cardiac Arrest? A Study from the Sonography in Cardiac Arrest and Hypotension in the Emergency Department (SHoC-ED) Investigators

39 Emergency department point-of-care ultrasound in outof-hospital and in-ED cardiac arrest

44 Impact of the modified SESAME ultrasound protoco implementation on patients with cardiac arrest in the emergency department

Peri-intubation cardiac arrest

11 Peri-Intubation Cardiac Arrest in the Pediatric Emergency Department: A Novel System of Care

31 Peri-intubation cardiac arrest in the Emergency Department: A National Emergency Airway Registry (NEAR) study

To perform bedside ultrasound in car-

35 Risk Factors for Peri-intubation Cardiac Arrest in a Pediatric Emergency Department

51 Factors Associated with the Occurrence of Cardiac Arrest after Emergency Tracheal Intubation in the Emergency Department diac arrest patients to differentiate true or pseudo-PEA and predict outcome

To investigate echocardiogram in life support use in cardiac arrest patients before and after a training day

Survey of physician's barriers to perform POCUS during cardiac arrest

To evaluate the predictive value of cardiac standstill visualized by bedside ultrasound in cardiac arrest

To perform bedside ultrasound in PEA/asystole cardiac arrest patients and compared to ECG rhythm

Evaluate whether detection of cardiac activity by ultrasound during ACLS is associated with improved survival

Evaluate the impact of a new ultrasoun protocol on patients with cardiac arrest

Evaluate a novel care system to mitigate risk for peri-intubation cardiac arrest in pediatric patients

To determine the incidence and clinica characteristics of peri-intubation cardiac arrest

The type of cardiac activity recorded during the ultrasound of the cardiac arrest patient might be related to the ROSC and survival at discharge prognosis

Use of echocardiogram in life support significantly increased after the training day

Top attending physician barriers relate to POCUS education, and the top resident physician barriers relate to logistics and the machines

Patients presenting with cardiac standstill on bedside echocardiogram do not survive to leave the ED regardless of their electrical rhythms

ECG rhythm alone is not an accurate predictor of cardiac activity and use of ultrasound may help to identify patients with ongoing mechanical cardiac activity

POCUS during cardiac arrest can identify patients with higher likelihood of survival to hospital discharge and can identify interventions outside of the standard ACLS algorithm

No significant survival benefits associated with the implementation of the modified SESAME protocol

A novel system of care mitigates the risk of peri-intubation cardiac arrest in pediatric patients in ED

Peri-intubation cardiac arrest for patients undergoing endotracheal intubation in the ED is rare. Higher likelihood of arrest occurs in patients with pre-intubation shock or hypoxemia

Hypoxia was the strongest predictor for peri-intubation cardiac arrest among children after emergent endotracheal intubation in pediatric patients receiving emergen intubation

Evaluate the incidence and clinical factors of peri-intubation cardiac arrest 
TA B L E 2. Continued.

\section{Extracorporeal CPR (ECPR)}

13 Managing Cardiac Arrest with Refractory Ventricular Fibrillation in the Emergency Department: Conventional Cardiopulmonary Resuscitation versus Extracorporeal Cardiopulmonary Resuscitation

24 Experience of Extracorporeal Cardiopulmonary Resuscitation in a Refractory Cardiac Arrest Patient at the Emergency Department

42 Predictors of Survival Following Extracorporeal Cardiopulmonary Resuscitation in Patients with Acute Myocardial Infarction-Complicated Refractory Cardiac Arrest in the Emergency Department: A Retrospective Study

Targeted Temperature Management

2 Perceived Barriers to Therapeutic Hypothermia for Patients Resuscitated from Cardiac Arrest: A Qualitative Study of Emergency Department and Critical Care Workers

16 Safety and Feasibility of Nasopharyngeal Evaporative Cooling in the Emergency Department Setting in Survivors of Cardiac Arrest

50 The Outcomes of Targeted Temperature Management After Cardiac Arrest at Emergency Department: A RealWorld Experience in a Developing Country

ROSC, return of spontaneous circulation; POCUS, point-of-care ultrasound; ED, emergency department; CPR, cardiopulmonary resuscitation; ECPR, extracorporeal cardiopulmonary resuscitation; ACLS, Advanced Cardiac Life Support; ECMO, extracorporeal membrane oxygenation; TTM, targeted temperature management.
Patients with refractory ventricular fibrillation receiving ECPR had a trend toward higher survival rates and significantly improved neurological outcomes

tion managed with conventional $\mathrm{CPR}$ or ECPR

To analyze the associated factors related to outcome and the post-ECPR managemen in patients who received ECPR due to nonresponse to advanced cardiac life support

To identify the determinant factors for clinical outcomes and survival rates of patients with cardiac arrest concurrent with acute myocardial infarction who underwent ECPR

To identify the barriers to implementation of mild therapeutic hypothermia for adult survivors of cardiac arrest

To demonstrate safety, feasibility and effectivity of nasopharyngeal evaporative cooling in comatose patients after successful resuscitation from cardiac arrest

To evaluate real-world practices of TTM To evaluate real-world pra

Early transition from ACLS to ECPR may improve the ECPR outcomes

ECMO insertion within 60 min of the arrival of patients with acute myocardial infarction and cardiac arrest at the ED increased the survival rate

The systematic adoption therapeutic hypothermia is met with interdependent generic, local, and individual barriers

Nasopharyngeal evaporative cooling used for one hour in primary cardiac arrest survivors is feasible and safe at flow rates of $40-50 \mathrm{~L} / \mathrm{min}$ in a hospital setting

TTM can improve survival and favorable neurological outcome in postcardiac arrest patients regardless of initial rhythm 


\section{Conclusions}

Studies focusing on EDCA are increasing but still scarce. The patient characteristics, etiology and outcome of EDCA are distinct from those of OHCA and IHCA and should be considered a unique group. Applying the knowledge arising from OHCA or IHCA to EDCA is questionable, and investigations focusing on EDCA are needed. Further studies evaluating the impact of prediction models in real world when applying with current risk stratification systems (ex: traditional triage system, intermittent vital sign monitoring) to avoid occurrence of EDCA are necessary. Developing skills applied in EDCA patients including ultrasound and peri-intubation protocol may improve outcome in specific subgroup and need further investigations. ED overcrowdedness and its relationship with EDCA is an important issue and developing a multifactorial, dynamic, individualized index to evaluate overcrowdedness is warranted for further study.

\section{AUTHOR CONTRIBUTIONS}

Conceptualization-HYL, CJN and SYC; Data curationHYL and SKH; Formal analysis - $\mathrm{CJN}$ and SYC; Figure preparation-SKH; Investigation-SYC, HYL and SKH; Writing-original draft-HYL and SKH; Writing-review \& editing-SYC and CJN. All authors read and approved the final manuscript.

\section{ETHICS APPROVAL AND CONSENT TO PARTICIPATE}

The Chang Gung Medical Foundation Institutional Review Board approved this study (IRB number: 201901062B0).

\section{ACKNOWLEDGMENT}

This research was supported by Chang-Gung Memorial Hospital. We are thankful to our colleagues who provided their expertise, which greatly assisted the research, although they may not agree with all the interpretations provided in this paper.

\section{FUNDING}

This research received no external funding.

\section{CONFLICT OF INTEREST}

The authors declare no conflict of interest. Shou-Yen Chen is the Guest Editor of the journal.

\section{SUPPLEMENTARY MATERIAL}

Supplementary material associated with this article can be found, in the online version, at https://oss. signavitae. $\mathrm{com} / \mathrm{mre}$-signavitae/article/1438784628250820608/ attachment/Supplementary\%20material. docx.

\section{DATA AVAILABILITY STATEMENT}

The datasets generated and/or analysed during the current study are available from the corresponding author on reasonable request.

\section{REFERENCES}

[1] Jacobs I, Nadkarni V, Bahr J, Berg RA, Billi JE, Bossaert L, et al. Cardiac arrest and cardiopulmonary resuscitation outcome reports: update and simplification of the Utstein templates for resuscitation registries: a statement for healthcare professionals from a task force of the International Liaison Committee on Resuscitation (American Heart Association, European Resuscitation Council, Australian Resuscitation Council, New Zealand Resuscitation Council, Heart and Stroke Foundation of Canada, InterAmerican Heart Foundation, Resuscitation Councils of Southern Africa). Circulation. 2004; 110: 3385-3397.

[2] Høybye M, Stankovic N, Holmberg M, Christensen HC, Granfeldt A, Andersen LW. In-Hospital vs. Out-of-Hospital Cardiac Arrest: Patient Characteristics and Survival. Resuscitation. 2021; 158: 157-165.

[3] Moskowitz A, Holmberg MJ, Donnino MW, Berg KM. In-hospital cardiac arrest: are we overlooking a key distinction? Current Opinion in Critical Care. 2018; 24: 151-157.

[4] Nolan JP, Soar J, Smith GB, Gwinnutt C, Parrott F, Power S, et al. Incidence and outcome of in-hospital cardiac arrest in the United Kingdom National Cardiac Arrest Audit. Resuscitation. 2014; 85: 987992.

[5] Kayser RG, Ornato JP, Peberdy MA; American Heart Association National Registry of Cardiopulmonary Resuscitation. Cardiac arrest in the Emergency Department: a report from the National Registry of Cardiopulmonary Resuscitation. Resuscitation. 2008; 78: 151-160.

[6] Gräsner JT, Herlitz J, Tjelmeland IBM, Wnent J, Masterson S, Lilja G, et al. European Resuscitation Council Guidelines 2021: Epidemiology of cardiac arrest in Europe. Resuscitation. 2021; 161: 61-79.

[7] Tricco AC, Lillie E, Zarin W, O'Brien KK, Colquhoun H, Levac D, et al. PRISMA Extension for Scoping Reviews (PRISMA-ScR): Checklist and Explanation. Annals of Internal Medicine. 2018; 169: 467-473.

[8] Munn Z, Peters MDJ, Stern C, Tufanaru C, McArthur A, Aromataris E. Systematic review or scoping review? Guidance for authors when choosing between a systematic or scoping review approach. BMC Medical Research Methodology. 2018; 18: 143.

[9] Valderrama AL, Fang J, Merritt RK, Hong Y. Cardiac arrest patients in the emergency department-National Hospital Ambulatory Medical Care Survey, 2001-2007. Resuscitation. 2011; 82: 1298-1301.

[10] Tan SC, Leong BS. Cardiac arrests within the emergency department: an Utstein style report, causation and survival factors. European Journal of Emergency Medicine. 2018; 25: 12-17.

[11] Mitchell OJL, Edelson DP, Abella BS. Predicting cardiac arrest in the emergency department. Journal of the American College of Emergency Physicians Open. 2020; 1: 321-326.

[12] Lee MJ, Ryu JH, Min MK, Lee DS, Yeom SR, Bae BK, et al. Predictors of survival and good neurological outcomes after in-hospital cardiac arrest. Signa Vitae. 2021; 17; 67-76.

[13] Chang Y, Shih H, Chen C, Chen W, Huang F, Muo C. Association of sudden in-hospital cardiac arrest with emergency department crowding. Resuscitation. 2019; 138: 106-109.

[14] Ye S, Liu J, He Y, Cao Y. Emergency department crowding might not strongly associated with higher incidence of in-hospital cardiac arrest. Resuscitation. 2019; 140: 72-73.

[15] Kim JS, Bae HJ, Sohn CH, Cho SE, Hwang J, Kim WY, et al. Maximum emergency department overcrowding is correlated with occurrence of unexpected cardiac arrest. Critical Care. 2020; 24: 305.

[16] Tsai L, Chien W, Chen C, Tsai S, Chaou C, Weng Y, et al. Association of patient-to-emergency department staff ratio with the incidence of cardiac arrest: A retrospective cohort study. Signa Vitae. 2021; 17; 118-124.

[17] Hwang U, McCarthy ML, Aronsky D, Asplin B, Crane PW, Craven CK, et al. Measures of crowding in the emergency department: a systematic review. Academic Emergency Medicine. 2011; 18: 527-538.

[18] Asplin BR, Magid DJ, Rhodes KV, Solberg LI, Lurie N, Camargo CA 
Jr. A conceptual model of emergency department crowding. Annals of Emergency Medicine. 2003; 42: 173-180.

[19] Jones P, Wells S, Ameratunga S. Towards a best measure of emergency department crowding: Lessons from current Australasian practice. Emergency Medicine Australasia. 2018; 30: 214-221.

[20] Wang A, Fang C, Chen S, Tsai S, Kao W. Periarrest Modified Early Warning Score (MEWS) predicts the outcome of in-hospital cardiac arrest. Journal of the Formosan Medical Association. 2016; 115: 76-82.

[21] Kim I, Song H, Kim HJ, Park KN, Kim SH, Oh SH, et al. Use of the National Early Warning Score for predicting in-hospital mortality in older adults admitted to the emergency department. Clinical and Experimental Emergency Medicine. 2020; 7: 61-66.

[22] Srivilaithon W, Amnuaypattanapon K, Limjindaporn C, Imsuwan I, Daorattanachai K, Dasanadeba I, et al. Predictors of in-hospital cardiac arrest within $24 \mathrm{~h}$ after emergency department triage: a case-control study in urban Thailand. Emergency Medicine Australasia. 2019; 31: 843-850.

[23] Lee SB, Kim DH, Kim T, Kang C, Lee SH, Jeong JH, et al. Emergency Department Triage Early Warning Score (TREWS) predicts in-hospital mortality in the emergency department. American Journal of Emergency Medicine. 2020; 38: 203-210.

[24] Awan SE, Sohel F, Sanfilippo FM, Bennamoun M, Dwivedi G. Machine learning in heart failure: ready for prime time. Current Opinion in Cardiology. 2018; 33: 190-195.

[25] Delahanty RJ, Alvarez J, Flynn LM, Sherwin RL, Jones SS. Development and Evaluation of a Machine Learning Model for the Early Identification of Patients at Risk for Sepsis. Annals of Emergency Medicine. 2019; 73: 334-344.

[26] Than MP, Pickering JW, Sandoval Y, Shah ASV, Tsanas A, Apple FS, et al. Machine Learning to Predict the Likelihood of Acute Myocardial Infarction. Circulation. 2019; 140: 899-909

[27] Wu C, Hsu W, Islam MM, Poly TN, Yang H, Nguyen PA, et al. An artificial intelligence approach to early predict non-ST-elevation myocardial infarction patients with chest pain. Computer Methods and Programs in Biomedicine. 2019; 173: 109-117.

${ }^{[28]}$ Lee S, Mohr NM, Street WN, Nadkarni P. Machine Learning in Relation to Emergency Medicine Clinical and Operational Scenarios: An Overview. The Western Journal of Emergency Medicine. 2019; 20: 219227.

[29] Ong MEH, Lee Ng CH, Goh K, Liu N, Koh ZX, Shahidah N, et al. Prediction of cardiac arrest in critically ill patients presenting to the emergency department using a machine learning score incorporating heart rate variability compared with the modified early warning score. Critical
Care. 2012; 16: R108.

[30] Liu N, Koh ZX, Goh J, Lin Z, Haaland B, Ting BP, et al. Prediction of adverse cardiac events in emergency department patients with chest pain using machine learning for variable selection. BMC Medical Informatics and Decision Making. 2014; 14: 75.

[31] Liu T, Lin Z, Ong MEH, Koh ZX, Pek PP, Yeo YK, et al. Manifold ranking based scoring system with its application to cardiac arrest prediction: a retrospective study in emergency department patients. Computers in Biology and Medicine. 2015; 67: 74-82.

${ }^{[32]}$ Hong S, Lee S, Lee J, Cha WC, Kim K. Prediction of Cardiac Arrest in the Emergency Department Based on Machine Learning and Sequential Characteristics: Model Development and Retrospective Clinical Validation Study. JMIR Medical Informatics. 2020; 8: e15932.

[33] Rajkomar A, Dean J, Kohane I. Machine Learning in Medicine. The New England Journal of Medicine. 2019; 380: 1347-1358.

[34] Liao SF, Chen PJ, Chaou CH, Lee CH. Top-cited publications on pointof-care ultrasound: The evolution of research trends. American Journal of Emergency Medicine. 2018; 36: 1429-1438.

[35] Mikati N, Callaway CW, Coppler PJ, Elmer J. Data-driven classification of arrest location for emergency department cardiac arrests. Resuscitation. 2020; 154: 26-30

[36] Ravindran R, Kwok CS, Wong CW, Siller-Matula JM, Parwani P, Velagapudi $\mathrm{P}$, et al. Cardiac arrest and related mortality in emergency departments in the United States: Analysis of the nationwide emergency department sample. Resuscitation. 2020; 157: 166-173.

[37] April MD, Arana A, Reynolds JC, Carlson JN, Davis WT, Schauer $\mathrm{SG}$, et al. Peri-intubation cardiac arrest in the Emergency Department: a National Emergency Airway Registry (NEAR) study. Resuscitation. 2021; 162: 403-411.

[38] Kim WY, Kwak MK, Ko BS, Yoon JC, Sohn CH, Lim KS, et al. Factors associated with the occurrence of cardiac arrest after emergency tracheal intubation in the emergency department. PLoS ONE. 2014; 9: e112779.

[39] Hoehn EF, Dean P, Lautz AJ, Frey M, Cabrera-Thurman MK, Geis GL, et al. Peri-Intubation Cardiac Arrest in the Pediatric Emergency Department: A Novel System of Care. Pediatric Quality \& Safety. 2020; 5: e365.

How to cite this article: Hsiang-Yun Lo, Shang-Kai Hung, ChipJin Ng, Shou-Yen Chen. Qualitative and quantitative analysis of emergency department cardiac arrest publications. Signa Vitae. 2022; 18(2): 78-87. doi:10.22514/sv.2021.209. 